\title{
Enabling Social Interaction through Real-Time Sketch-Based Communication
}

\author{
Nadir Weibel ${ }^{1}$, Lisa G. Cowan ${ }^{2}$, Laura R. Pina ${ }^{2}$,William G. Griswold ${ }^{2}$, James D. Hollan ${ }^{1}$ \\ ${ }^{1}$ Department of Cognitive Science, ${ }^{2}$ Department of Computer Science and Engineering \\ University of California, San Diego, 9500 Gilman Dr., La Jolla, CA 92093, USA \\ \{weibel, lgcowan, lrpina, wgg, hollan\}@ucsd.edu
}

\begin{abstract}
We present UbiSketch, a tool for ubiquitous real-time sketchbased communication. We describe the UbiSketch system, which enables people to create doodles, drawings, and notes with digital pens and paper and publish them quickly and easily via their mobile phones to social communication channels, such as Facebook, Twitter, and email. The natural paperbased social interaction enabled by UbiSketch has the potential to enrich current mobile communication practices.
\end{abstract}

ACM Classification: H.5.2 [Information Interfaces and Presentation]: User Interfaces - Input devices and strategies

General terms: Design, Human Factors

Author Keywords: Sketching, interactive paper, digital pen, mobile phone, communication, social networks

\section{INTRODUCTION}

With people constantly on the go and social communities spread out, people need quick, easy ways to exchange information. A range of applications on mobile devices now support communication nearly anywhere and anytime. Despite the need for fast access to these communication tools, the typical interaction workflow is tedious, demanding time and attention (taking the device out, switching it on, using an application, switching it off, putting it away).

In addition to the mobile phones, pens are still commonly carried, since they provide a simple way to make notes and annotate paper documents. The ubiquity of this pair of devices speaks to their usefulness. Paper documents have important properties that have yet to be achieved by digital media, including portability, flexibility, readability, and high resolution [7]. Pen and paper are widely used, especially in comparison to digital tools [8], for note taking and doodling during lectures and meetings, and informal communication via sketching. Paper-based interfaces that digitize input enable users to exploit the affordances of digital media (e.g., search, storage, modification and distribution) in a mixed paper-digital environment.

We present UbiSketch, a mixed paper-digital system integrating Anoto Digital Pen and Paper technology ${ }^{1}$ and 3G smart phones. UbiSketch exploits the affordances of pen and paper to support quick, easy, informal, sketch-based communication on mobile phones. We describe how the system enables

\footnotetext{
${ }^{1} \mathrm{http}: / / w w w . a n o t o . c o m$

Copyright is held by the owner/author(s).

UIST'10, October 3-6, 2010, New York, New York, USA.

ACM 978-1-4503-0271-5/10/10
}

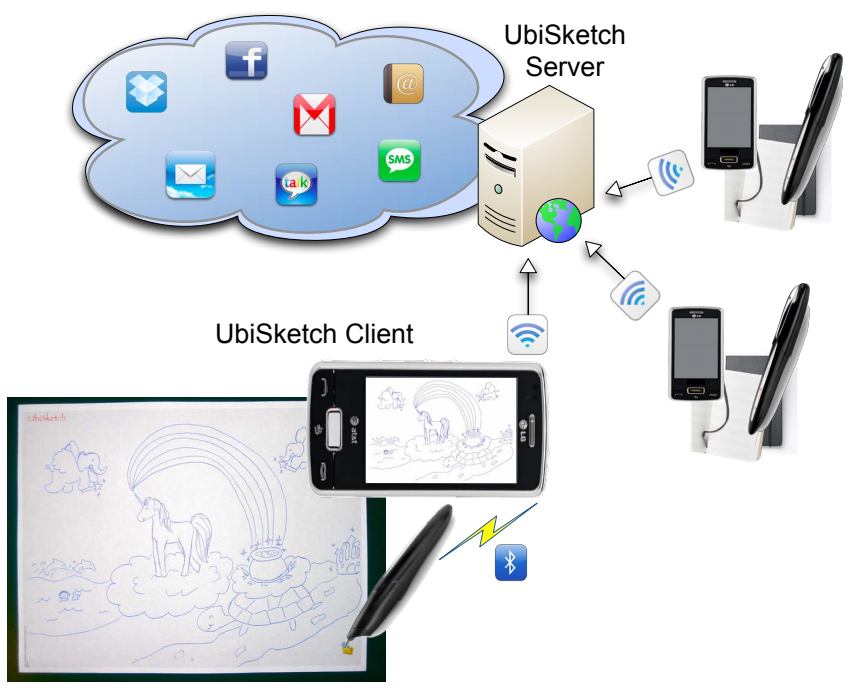

Figure 1: UbiSketch system overview

hand-written or drawn notes to be shared via social communication channels, such as Facebook, Twitter, or email.

\section{RELATED WORK}

As Buxton observed [1], sketches are quick to make, timely (provided when needed), inexpensive, disposable, and show the peculiarities of individuals' handwriting. Like spoken and written language, sketches are a form of communication with self and others, and they externalize ideas for remembering and sharing [10]. Although sketching with pen and paper is inexpensive, intuitive, and quick, paper-based sketches can be difficult to re-access, rearrange, or share [3]. Digital tablets or boards can enable digital input and interactive feedback $[9,5]$, yet the experience and feeling of sketching differs from that of sketching on paper, and users often have to adjust their drawing techniques accordingly [2]. Various research projects have demonstrated the effectiveness of digital pen and paper technology for harnessing the affordances of paper and digital media $[4,12,11]$.

\section{UBISKETCH}

UbiSketch is a mixed paper-digital infrastructure that tracks pen and paper sketches and transmits them in real-time to networked digital services. Users carry an Anoto digital pen, for capturing paper-based interactions, and a Bluetooth enabled smart phone, for connecting to the Internet. We are using Magicomm G303 and Anoto DP-201 digital pens, which can stream data in real time over Bluetooth to a separate 
digital device for further processing. The UbiSketch infrastructure, illustrated in Figure 1, is based on an extension of the iPaper framework [6] for Windows-based mobile smart phones. A lightweight UbiSketch client, running on a smart phone, processes and temporarily stores information streamed from the digital pen over a Bluetooth connection. When the user publishes the paper-based sketch, the UbiSketch client forwards the recorded information, augmented with supplementary data (current GPS position and ID of the phone, digital pen, and paper document used), to a server on the network. The UbiSketch server further processes the recorded pen strokes and supplementary data, and forwards the sketch to one or more digital services such as email, Facebook, Twitter, MMS or RSS.

Users interact with UbiSketch through paper documents, imprinted with the Anoto pattern. We offer different paper formats (small and large notebooks and loose sheets), to balance size and portability. Users can interact with UbiSketch by tapping with the pen on the printed "buttons" deployed on a paper control panel (Fig. 2). Via this control panel, users can save a sketch locally, clear the current sketch, load a previously-saved sketch, or publish a sketch to Facebook, Twitter, or the UbiSketch Diary (which emails the sketch to a pre-defined address). The control panel can be easily modified to support evolving functionality.
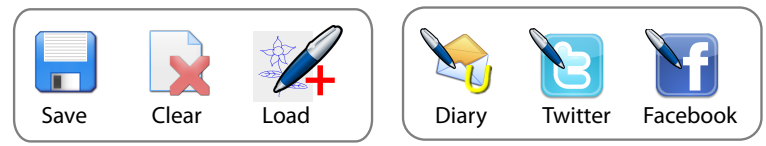

Figure 2: UbiSketch paper-based control panel

The UbiSketch server can publish paper-based sketches to any digital service on the Internet. The server has a flexible plug-in architecture, that enables the easy development and deployment of dedicated plug-ins for supporting additional publication channels. The channels presented in this demo (SketchBook and SketchTweet) are based on the facebookjava-api ${ }^{2}$ and Twitter $4 J^{3}$ libraries, and the system can easily be extended to support other channels. Sketchbook is a Facebook application that enables sketches to be posted directly to a user's "Wall". Subsequent social interactions, such as comments or "likes", are directly supported by the Facebook interface. SketchTweet provides similar functionality for publishing sketches to Twitter.

We ran an exploratory study, intended to inform our iterative design process, of UbiSketch usage in a controlled environment. We observed users' experiences publishing sketches to Facebook with a working prototype. Their published sketches, despite being created in a laboratory setting, led to conversation and social interaction on the Web and in person. Some sketches received many "likes" and comments on Facebook. Participants expressed interest in continuing to publish to Facebook and other channels (SMS, MMS, Twitter, RSS).

Our study also shed light on the user interface design. All participants reported that sketching felt natural, just like drawing with an ordinary pen on paper. They also preferred for

\footnotetext{
${ }^{2} \mathrm{http}: / /$ code.google.com/p/facebook-java-api

${ }^{3} \mathrm{http}: / /$ twitter4j.org
}

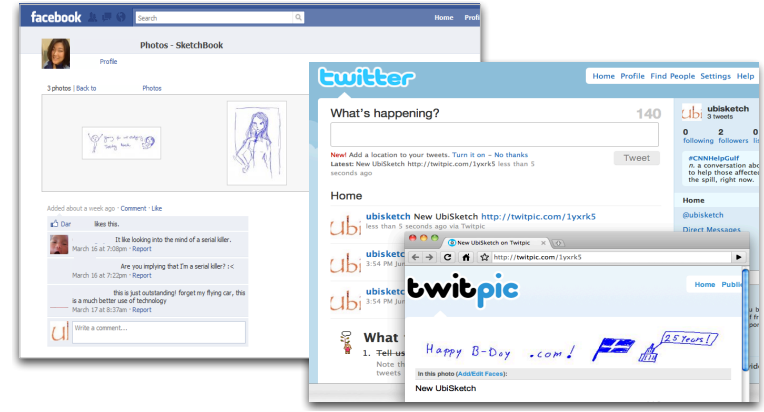

Figure 3: SketchBook / SketchTweet

the paper to be the primary interface, with the phone providing supplementary feedback.

\section{CONCLUSION}

UbiSketch enables people to publish paper-based sketches to online channels with minimal effort, in real-time. We leverage current practices for mobile communication and penand-paper interaction to create a resource for self-expression and informal social interaction. The infrastructure we have developed is extensible, and could easily support additional communication channels. UbiSketch shows promise as a mechanism for lightweight visual communication, exploiting the affordances of pen and paper, mobile phones, and online social networks. We plan to conduct a field study to observe naturalistic usage practices.

\section{ACKNOWLEDGMENTS}

This work has been supported by NSF grant 0729013 , UC MICRO/Microsoft Research grant 07-067 and Microsoft Research ER\&P.

\section{REFERENCES}

1. B. Buxton. Sketching User Experiences. M. Kaufmann, 2007.

2. C. H. Faber. Digital Drawing Tablet to Traditional Drawing on Paper: A Teaching Studio Comparison. In Proc. IASDR 2009.

3. J. A. Landay and B. A. Myers. Interactive sketching for the early stages of user interface design. In Proc. CHI '95, pages 43-50, 1995.

4. C. Liao, F. Guimbretière, K. Hinckley, and J. Hollan. PapierCraft: A Gesture-Based Command System for Interactive Paper. ACM TOCHI, 14(4):1-27, 2008.

5. G. Martin, J. Pittman, K. Wittenburg, et al. Sign Here, Please (Interactive Tablets). BYTE, 15(7):243-251, 1990.

6. M. C. Norrie, B. Signer, and N. Weibel. General Framework for the Rapid Development of Interactive Paper Applications. In Proc. CoPADD 2006.

7. A. J. Sellen and R. H. Harper. The Myth of the Paperless Office. MIT Press, 2003.

8. J. Steimle, I. Gurevych, and M. Mühlhäuser. Notetaking in University Courses and its Implications for eLearning Systems. In Proc. DeLFI 2007.

9. I. E. Sutherland. SketchPad: A Man-Machine Graphical Communication System. In Proc. AFIPS 1963.

10. B. Tversky. What do Sketches say about Thinking. Technical Report SS-02-08, AAAI, 2002.

11. N. Weibel, A. Ispas, B. Signer, and M. C. Norrie. PaperProof: A Paper-Digital Proof-Editing System. In Proc. CHI 2008.

12. R. B. Yeh, C. Liao, S. R. Klemmer, F. Guimbretière, B. Lee, B. Kakaradov, J. Stamberger, and A. Paepcke. ButterflyNet: A Mobile Capture and Access System for Field Biology Research. In Proc. CHI 2006. 